\title{
Homocysteine and Cardiac Neural Crest Cell Cytoskeletal Proteins in the Chick Embryo
}

\author{
Anthony R. Mwakikunga \\ Department of Biology, University of Northern Iowa, \\ Cedar Falls, Iowa, 50614, USA \\ Tel: 265884038909 Email: anthonymwakikunga@yahoo.com \\ Amanda L. Clubine \\ Department of Biology, University of Northern Iowa, \\ Cedar Falls, Iowa, 50614, USA \\ Tel: 319-939-4924Ｅ-mail:amandaclubine@isu.edu \\ Darrell J. Wiens (Corresponding author) \\ Department of Biology, University of Northern Iowa, \\ Cedar Falls, Iowa, 50614, USA \\ Tel: 319-273-6880Ｅ-mail: wiens@uni.edu
}

Received: January 15, 2011 Accepted: February 10, 2011 doi:10.5539/ijb.v3n2p43

The majority of this research was carried out in fulfillment of the requirements for the M.S. degree in the Department of Biology, University of Northern Iowa by A.M. This work was partially supported by a grant to A.M. from the College of Natural Sciences at the University of Northern Iowa, and by a summer undergraduate research award to A.C.

\begin{abstract}
Elevated serum homocysteine (Hcys) is correlated with cardiovascular disease and with embryonic malformations related to neural crest cells (NCCs). We predicted Hcys may alter the balance of actin networks, stress fibers and focal adhesions, altering migration. We cultured neural tube explants in control and Hcys-treated medium and visualized actin, $\alpha$-actinin, vinculin, filamin, and LIM3 protein in NCCs migrating outward. In Hcys, phalloidin-stained actin in stress fibers was brighter, and vinculin was more abundant in focal adhesions and lamellipodia. $\alpha$-actinin and LIM3 were also enhanced around nuclei and in focal adhesions, and $\alpha$-actinin also in filopodia. Filamin was unchanged. Hcys caused more spreading and migration of NCCs, but not more cell-cell adhesions. The findings support our hypothesis that Hcys adjusts NCCs for greater adhesion and migration. Its effect on LIM3 suggests it may modulate signaling that adjusts the cytoskeleton for enhanced migration, leading to mistimed and defective development of target tissues.
\end{abstract}

Keywords: Homocysteine, Chick embryo, Neural crest cells, Cytoskeleton, Ras GTPase, LIM3 protein

\section{Introduction}

Neural Crest Cells (NCCs) constitute a population of multipotent neuroectodermal cells in vertebrate embryos, originally found between the neural tube and epidermis. While the neural tube is forming, NCCs begin an epithelial-to-mesenchymal transition (EMT) by disengaging and remodeling their cell junctions, changing their shapes and reorganizing their cytoskeletons to become motility competent. NCCs then migrate to diverse destinations where they form a wide array of differentiated tissues. In the neural tube region of the rhombencephalon between the mid-otic placode and the third somite pair (Kirby et al., 1985; Kirby \& Hutson, 2010), NCCs migrate along circumpharyngeal pathways to reach the aortic arches and portions of their 
derivatives, importantly, the common carotid and brachiocephalic arteries and aortic arch (reviewed by Kirby \& Waldo, 1995). Here they enter and form the endothelium of the arteries. They also enter the cardiac outflow tract where they form the aorticopulmonary septum (Kirby et al., 1983; Poelmann \& Gittenberger-de Groot, 1999; Hutson \& Kirby, 2003; Kaartinen et al., 2004). In addition, these cardiac NCCs participate in forming the pharyngeal arches and their glandular and connective tissue derivatives. It has been suggested that these colonizing NCCs may play additional, unexpected roles during cardiovascular development. Studies in both mice and chick embryos suggest that cardiac NCCs can also enter the heart, not only its outflow region, but also the venous pole and dorsal mesocardium at a later time (Poelmann et al. 1998; Poelmann \& Gittenberger-de Groot, 1999). Some investigators have reported that NCCs contribute to the adult semilunar and atrioventricular valves as well as part of the conducting system of the heart (Solloway and Harvey, 2003; reviewed by Snider et al., 2007). Though this has been disputed (Gourdie et al., 2003), it is clear that cardiac NCCs have pivotal roles in cardiogenesis, and that any disruption of their behavior can be predicted to lead to neural crest associated heart defects, including persistent truncus arteriosus, tetralogy of Fallot, transposition of the great arteries, DiGeorge syndrome and interrupted aortic arches. Although these are among the less frequent defects, they are responsible for the vast majority of deaths from cardiac anomalies (Clark, 1996).

Assembly, disassembly and reorganization of actin and its binding partners must occur during EMT and subsequent migration of the cardiac NCCs. This complex process involves the participation and regulation of many molecules. Among them are LIM3 (also known as cysteine and glycine-rich protein 3 or CRP3), and the actin regulating Ras GTPases Rho, Rac, and cdc42, which control the deployment and dynamic activity of stress fibers, focal adhesions, lamellipodia and filopodia in migrating cells. LIM3 is found mainly in the cytosolic and sarcomeric components of cardiomyocytes (Arber et al., 1997), however it is also cytoskeletal (Flick and Konieczny, 2000). It functions as an F-actin binding protein involved in actin cytoskeleton remodeling (reviewed by Khurana et al., 2002; Zheng et al., 2008). LIM3 is also involved in focal adhesions where focal adhesion kinase (FAK) and paxillin, a substrate for FAK, co-localize. Paxillin actually contains a LIM3 domain, and functions as an adaptor, responsible for the recruitment of structural and signaling molecules to focal adhesions, as has been described to occur in fish fibroblasts (Brown et al., 1996). Cytoskeletal localization studies in which smooth muscle tissues transfected with plasmids encoding a paxillin mutant having a deletion of the LIM3 domain (paxillin LIM3 dl 444-494, a primary determinant for targeting paxillin to focal adhesions), have demonstrated that recruitment of paxillin and vinculin to the smooth muscle membrane is necessary for tension development. These studies showed that recruitment of vinculin to the membrane is regulated by paxillin, and that paxillin recruitment is mediated by its LIM3 domain (Saez et al., 2004). This supports earlier studies, which revealed that the principal mechanism of targeting paxillin to focal adhesions is through LIM3 (Brown et al., 1998). In this manner, LIM3 together with vinculin, another focal adhesion protein may participate in regulating the formation of linkages between the cytoskeleton and integrin receptors that mediate tension transmission between the contractile apparatus and the extracellular matrix during muscle contraction. Any inhibition of LIM3 would prevent paxillin and vinculin recruitment to the membrane, and would inhibit the development of force. Therefore, LIM3 is essential in focal adhesion formation and function.

In order to have a direct mechanical coupling between integrins and the actin cytoskeleton, the focal adhesion proteins talin and $\alpha$-actinin must also link the cytoplasmic domain of $\beta$-integrins with actin filaments. Vinculin, acting as another linker protein, also binds actin filaments, talin and $\alpha$-actinin (Critchley et al., 1999). In order to support force transfer from the cytoskeleton to the extracellular matrix vinculin strengthens connections between integrins and actin filaments (Choquet et al., 1997). Other studies have provided evidence that the binding of vinculin to actin and talin can be regulated by phosphatidyl-inositol-4-5-bisphosphate (Gilmore \& Burridge, 1996) and inhibited by acidic phospholipids (Weekes et al., 1996). Clearly the structural links between actin filaments and integrins are regulated in some cell types, and it would not be unreasonable to hypothesize that agents known to alter cell migration may do so by affecting the expression or function of LIM3 protein, which is essential in actin binding as well as in focal adhesion dynamics.

Remarkably, LIM3 is also a transcription factor that is crucial in embryogenesis, particularly known for its role in the development of the pituitary (Pogoda et al., 2006; Sjodal \& Gunhaga, 2008) and the retina (Edqvist et al., 2006). A mutation of it has been implicated in human hypertrophic cardiomyopathy (Maron et al., 2000). With regard to migratory cardiac NCCs, a microarray analysis study of homocysteine-responsive genes in these cells has shown that there is a modest down-regulation of seventeen out of nineteen cell migration or adhesion related genes, but a striking 177-fold up-regulation of LIM3 expression (Rosenquist et al., 2007).

Homocysteine (Hcys) is an amino acid found in blood, first described by Butz and du Vignead (1932), and first linked to human disease by Carson \& Neil (1962). Elevated Hcys levels in maternal blood and amnionic fliud 
have been implicated in the cause of not only cardiovascular (Kapusta et al., 1999), but also renal (Bostom \& Lathrop, 1997) and skeletal and endocrine diseases. They are also implicated in spontaneous abortion, placental abruption, low infant birth weight, osteoporosis, and pre-menstrual syndrome (reviewed by Nelen et al., 1997; Refsum et al., 2006). Furthermore, elevated Hcys levels are implicated in neural tube defects (Steegers-Theunissen et al., 1991; Mills et al., 1996; Epeldegui et al., 2002), although some investigators have reported the failure of Hcys to induce neural tube defects in mice (Bennette et al., 2006). Thus Hcys's teratogenicity could be species dependent. Multiple mechanisms of action of Hcys in altering NCC behavior are known, and these manifest mainly in modes that include cell proliferation (Dalton et al., 1997; Tierney et al., 2004;), apoptosis (Brauer \& Tierney, 2004; Sabapathy et al.,1999), extracellular protease activity (Giambernardi et al., 2001; Cai and Brauer, 2002; Yang and Zou, 2003), NMDA receptor ligand binding (Andale et al., 1998; Rosenquist et al., 1996; Rosenquist et al., 1999), and EMT/cell migration (Moephuli et al., 1997; Wang, 1999; Tierney et al., 2004; Heidenreich et al., 2008). There is some evidence of yet other mechanisms (Liu et al., 1997; Raposo et al., 2004; Loscalzo J., 1996; Wang, 1999). Clearly, Hcys has diverse mechanisms of action in altering cell behavior, but its demonstrated effects on the migratory behavior of NCCs are provocative. It has been shown to increase migration distance and cell motility (Brauer \& Rosenquist, 2002), and also outgrowth area of NCCs in culture (Boot et al., 2003; Boot et al., 2004a). It can encourage EMT (Boot et al, 2003). Its striking elevation of LIM3 expression in cardiac NCCs in vitro (Rosenquist et al., 2007) together with the comprehensive capabilities of LIM3 to activate developmental genes and remodel the actin cytoskeleton and focal adhesions, lead us to hypothesize that Hcys acts via the LIM3 protein to alter NCC motility. We predicted that evidence of this would be found in the levels and localization of LIM3 and key proteins of the stress fibers, focal adhesions, and lamellipodia in Hcys-treated NCCs.

\section{Materials and methods}

\subsection{Embryos and Neural Tube Explant Culture}

Fertile hybrid strain (Black Astralorps crossed with New Hampshire Red) chicken eggs were purchased from Sunray Hatchery, Hazelton, IA. Embryos at stages 10 to 13 (Hamburger and Hamilton, 1951) were removed from $36-44 \mathrm{hr}$ incubated eggs by placing $3 \mathrm{MM}$ filter paper rings over them and cutting around the perimeter. The embryos were placed in sterile Chick Ringer's saline in plastic dishes. Glass needles were used to dissect out cardiac neural tubes from the level of mid-otocyst to the third somite pair. The neural tubes were then placed into Falcon Primaria culture dishes containing $2 \mathrm{ml}$ of Medium 199 with $10 \%$ fetal bovine serum (FBS) and 1\% penicillin/streptomycin. To experimental dishes 2 or $6 \mu \mathrm{L}$ ( 6 only for some phalloidin staining experiments) of $100 \mathrm{mM}$ Hcys was added to $2 \mathrm{ml}$ of medium (final concentration: $100 \mu \mathrm{M}$ or $300 \mu \mathrm{M}$ ). Concentrations of Hcys in the $100 \mu \mathrm{M}$ range are known to be effective in ovo as well as in vitro (Rosenquist et al., 1996; Brauer and Rosenquist, 2002; Boot et al., 2003; Tierney et al., 2004; Heidenreich et al., 2008). The control dishes received $2 \mu \mathrm{L}$ of Chick Ringer's only. The culture dishes were incubated at $38^{\circ} \mathrm{C}$ in $5 \% \mathrm{CO}_{2}$ and removed approximately $24 \mathrm{hr}$ later. Only attached explants were used for study.

\subsection{Actin staining and intensity measurement}

Neural tube explants were washed in phosphate buffered saline (PBS) and then fixed in 3.7\% formaldehyde in PBS for $10 \mathrm{~min}$. They were then washed in PBS and permeabilized $30 \mathrm{~min}$ in $0.1 \%$ TritonX-100 in PBS. Each explant was stained in the dark for F-actin by incubating in $100 \mu \mathrm{L}$ of a $5 \mu \mathrm{g} / \mathrm{mL}$ solution of fluorescein isothiocyanate (FITC) labeled phalloidin (Sigma Chemical Company, St. Louis, MO) in 1\% dimethyl sulfoide (DMSO) in PBS. They were then washed in PBS and mounted in anti-fade mounting medium under a coverslip. For analysis, the explants were viewed on a Leica DMIRE inverted fluorescence microscope and images were captured using an Optronics CCD digital camera and MagnaFire software (Optronics, Inc., Goletta, CA). In regions of migratory cells that showed high stain resolution the staining fluorescence intensity was evaluated using Image $\mathbf{J}$ software by drawing a single line across each cell and using the histogram tool to measure brightness on a 0-256 scale. All microscope, camera and computer settings were kept constant. Phalloidin-stained individual cell areas were also measured from randomly chosen cells, those that were in contact with a grid of twelve lines drawn radially from the explant center out to the perimeter of the area of migratory cells. Averages for Hcys-treated (observed) and control (expected) cultures were compared for statistical differences using a Student's T-test.

\subsection{Immunolocalization}

Explants to be evaluated for immunostaining were fixed in ice-cold 20\% DMSO in methanol for $1 \mathrm{hr}$. After fixation explants were washed 3 times, 2 min each in PBS. This was followed by a 10 min peroxidase quenching solution treatment in $30 \% \mathrm{H}_{2} \mathrm{O}_{2}$ in methanol, and then 3 washes in PBS. Explants were then permeabilized for 30 
min in $0.1 \%$ Triton X-100 in PBS, and incubated in blocking solution $(0.5 \%$ bovine serum albumin, $1 \%$ Tween-20, 1\% non-fat dry milk in PBS) for 30 min at ambient temperature. Avian monoclonals anti- $\alpha$-actinin (1E12) and anti-vinculin (VN 3-24) from the Developmental Studies Hybridoma Bank (DSHB), Iowa City, and mouse monoclonal anti-filamin A (PM6/317) from the Millipore Corporation (Temecula, CA) were diluted 1:75 in blocking solution. Monoclonal anti-LIM3 antibody purchased from the DSHB was diluted 1:5 in blocking solution made up with 3\% bovine serum albumin and 0.3\% Triton X-100 in PBS, pH 7.4 (PBSAT). The explants were incubated in primary antibody for $30 \mathrm{~min}$ at room temperature or for $24 \mathrm{hr}$ at $4{ }^{\circ} \mathrm{C}$. This was followed by 3 PBS washes, 2 min each for anti- $\alpha$-actinin and anti-vinculin, but 5 min each following anti-LIM3 incubations. The binding of anti- $\alpha$-actinin and anti-vinculin was detected using a biotinylated secondary antibody (incubated $30 \mathrm{~min}$ ) and strepavidin-peroxidase conjugate $(10 \mathrm{~min})$ from a Zymed-Invitrogen Histostain kit (S. San Francisco, CA). To detect anti-LIM3 binding, the explants were incubated with biotinylated anti-mouse IgG diluted 1:100 in PBSAT for $2 \mathrm{hr}$ at ambient temperature. Chromagen mixture from the kit was then applied to the dishes and incubated in darkness for $10 \mathrm{~min}$ to develop color. The explants were then rinsed in deionized water to stop color development and post-fixed in $3.7 \%$ formaldehyde, and were stored at $4{ }^{\circ} \mathrm{C}$. For immunolocalization of LIM3 in embryos, whole embryos were fixed, blocked, and incubated in anti-LIM3 as with explants. After washing 3 times in PBS, Alexaflour 488 tagged anti-mouse IgG $(2 \mathrm{mg} / \mathrm{ml}$, Molecular Probes, Inc., Eugene, OR) diluted 1:500 in blocking solution was added to the dishes with whole embryos to detect the primary antibody for 30 minutes at ambient temperature. Embryos were then washed in PBS for 15 minutes and stored in $70 \%$ Ethanol.

\subsection{Photography and image analysis}

Images of immunostained cells were taken at automatic exposure using a Leica DMIRE2 inverted microscope, Q-Imaging QI Fast 1394 camera, and IPLAB (Scanalytics, Inc., Fairfax, VA) and MagnaFire software (Optronics, Inc., Goleta, CA). To randomly sample cultured cardiac NCCs we drew a 12-line radial grid over color images of immunostained neural tube explants, and then evaluated the set of cells intersected by the lines for color immunostaining intensity (protein expression), cell morphology and cell-cell attachment. The cells in each culture were scored as having high, medium or low color intensity. In addition, we used IPLAB software to quantitate staining via color thresholding for all cultures. The images were opened in IPLAB and the segmentation function was used to measure the area of red above the threshold. To achieve uniform thresholding, each image was indexed for grayscale brightness (scale $=0-256)$ to determine the minimum threshold level prior to measuring red pixels above threshold. For consistency, all images were set at a minimum brightness of 115 . The red above threshold from within the explant was excluded in order to evaluate only cardiac NCCs that had migrated out from the explant. The red pixels above threshold level were converted to $\mu \mathrm{m}^{2}$ according to software calibration. The data (means) for Hcys-treated (observed) and control (expected) cultures were compared for statistical differences using a Student's T-test. In total, 217 explant cultures were imaged, counted and thresholded. Whole embryos stained fluorescently for anti-LIM3 were photographed on the stage of a Leica MZ16 fluorescence stereomicroscope using the Optronics CCD digital camera and MagnaFire software.

\section{Results}

When explants from the cardiac segment of the neural tube were explanted to Primaria culture dishes they attached quickly and cells migrated outward rapidly to form an expanding area of cells by $24 \mathrm{hr}$ in culture. There were two populations of spreading cells. Neuroepithelial cells spread outward as a sheet of interconnected, flat cells. However, the NCCs migrated outward individually and in small clusters. We assessed the arrangement in NCCs of F-actin, LIM3 protein, $\alpha$-actinin, vinculin, and filamin in the presence or absence of $100 \mu \mathrm{M}$ Hcys. We also assessed F-actin in the presence of $300 \mu \mathrm{M}$ HCys.

\subsection{F-actin}

To visualize the actin cytoskeleton in 24-hr Hcys-treated and control cardiac NCC cultures we fixed, permeabilized and then stained with FITC-labeled phalloidin to visualize the actin cytoskeleton and also measured the fluorescence intensity. Hcys-treated migratory cells appeared to have more prominent stress fibers than untreated cells, and the effect was more vivid in $300 \mu \mathrm{M}$ Hcys than in $100 \mu \mathrm{M}$ (Fig. 1, A-C). Following measurements using Image $\mathbf{J}$ software, we found that the cytoskeletal actin fluorescence intensity in individual cells averaged $20 \%$ of maximum possible intensity in untreated cultures, but showed an enhancement of $8 \%$ for $100 \mu \mathrm{M}$ Hcys-treated cultures and $24 \%$ for $300 \mu \mathrm{M}$ Hcy-treated cultures, both significant increases, confirming its effects on actin infrastructure (Fig. 1, D). Actin staining was affected in this way in migratory cells but not in epithelial layer cells. Migratory cell area and shape were not visibly affected by Hcys treatment. 


\subsection{LIM3 protein}

Immunolocalization by peroxidase staining (red) showed that the LIM3 Protein was evident mainly in the nuclei and focal adhesions of migratory cells, although there was also faint staining throughout the cytoplasm (Fig. 2, A, control, and B, Hcys). The nuclei and focal adhesions appeared to be slightly more intense in Hcys-treated cells. Color thresholding measurements of red stain from images of immunostained cultures with each of the antibodies are displayed in Table 1. The results of this quantitation for LIM3 protein revealed a significantly higher level in the Hcys-treated cultures than in controls $(\mathrm{p}<0.026)$. We also used whole embryo immunolocalization of LIM3 in order to verify cardiac NCC localization and identify other tissues where it is prominent. We found that it was more strongly expressed in the rostral extent of the head and in the heart than in other regions (Fig. 2, D, treated, and E, control). Moderate staining was evident in the optic cup and the mandibular and hyoid arches. There was a narrow strip of brighter stain visible midway between the sides of the conotruncus and aligned with its long axis, where the aorticopulmonary septum would be expected (Fig. 2, D).

\subsection{Immunolocalization $\alpha$-actinin, vinculin, and filamin}

$\alpha$-actinin was most strongly expressed in filapodia and just outside of the nuclei of most migrating NCCs in explant cultures (Fig. 3, A and B). It also stained stress fibers and focal adhesions, especially in Hcys-treated cultures (Fig. 3, B). Its level was $77 \%$ higher than in migratory cells of the control cultures, which is apparent from Fig. 3, A and B, and which is evident from color thresholding, where we found a significant difference (Table 1).

Immunostaining for vinculin revealed intense staining of focal adhesions and also moderate staining of lamellipodia (Fig. 3, C and D). The vinculin-stained focal adhesions appeared larger and more prominent than those stained with anti- $\alpha$-actinin, and the staining was more intense in treated cultures than in control cultures. It was more than twice as extensive when measured by color thresholding, a significant difference (Table 1). Even for cells stained at medium pixel intensity levels there was a significantly greater mean number of square microns thresholded in Hcys-treated cultures $(\mathrm{p}<0.001)$.

Because migrating cells use filamin to create networks of actin in leading lamellae when Rac is activated, we also investigated whether Hcys alters its expression. As expected, anti-filamin immunostaining was observed along the cell periphery of cultured cells (Fig. 3, E and F). It showed more intense staining in the presence of Hcys as well. However, the difference was not statistically significant (Table 1). Interestingly, there were more intense, stained cavities or vesicles in treated than in control cultures as can be seen in Figure 3, although this difference was also not statistically significant. These had the approximate dimensions of caveolae (larger) and trans-Golgi vesicles (50-100 nm diameter). A summary of our results for immunolocalization of $\alpha$-actinin, vinculin, filamin and LIM3 proteins, and of their functions is shown in Table 3.

\subsection{Cell morphology and cell-cell attachment pattern}

To reveal the effects of Hcys on the morphology and cell-cell attachment pattern of cultured cardiac NCCs, we used a software-generated twelve radial line array to randomly sample cells. The sampled cells were categorized as either spread or round, and attached to another cell or not attached. The data (mean numbers) for Hcys-treated (observed) and control (expected) cultures were compared using the Student's T-test. The data are shown in Table 2. It was apparent that the Hcys-treated cultures had significantly more spread cells with irregular, migratory shape than control cultures. However, no significant difference was found regarding the amount of cell-cell attachment between Hcys-treated and control cultures.

\section{Discussion}

Cells that are migrating need to organize their Ras GTPases at the appropriate time and place. Where Rho is activated, actin is arranged along with myosin II and other proteins into parallel bundles cross-linked by $\alpha$-actinin to form stress fibers, and these insert into focal adhesions, which contain the linker proteins vinculin and talin, among others. Migrating cells use stress fibers for contraction and maintenance of tension in conjunction with focal adhesions, which act as anchor points to mediate cell shape dynamics and migration. Similarly, Rac and cdc42 must be regulated so that cells can dynamically organize the actin cytoskeleton for the assembly and coordinated use of lamellapodia and filapodia for extension and other aspects of motility.

Heidenreich et al. (2008) showed that Hcys enhances attachment in cultured chick cardiac NCCs, and that vinculin-positive focal attachment sites were more numerous and F-actin bundles were thicker and longer. They indirectly demonstrated that these effects are brought about by increases in intracellular calcium ion levels. We have provided additional evidence that Hcys enhances actin-based stress fibers and that the effect is enhanced at a three-fold higher concentration $(300 \mu \mathrm{M})$. We have also confirmed an increase in vinculin presence in focal 
adhesions. Vinculin promotes focal adhesion formation by interacting directly with talin and actin (Humphries, et al., 2007). The more focal adhesion proteins become organized, the more they are linked to integrins, hence linking migrating cells to the extracellular matrix for attachment. In this study we showed that the presence of Hcys was correlated with an increase in vinculin expression in focal adhesions and lamellapodia of flat migrating cells. Vinculin immunostaining was more intense in treated cultures where focal adhesions were larger and more numerous (Fig. 3, D) than in control cultures (Fig. 3, C). We confirmed this by using color-thresholded area measurements, which showed an increase of $125 \%$ (Table 1). Thus Hcys-treated cardiac NCCs deploy more vinculin to the membrane to make focal adhesions, enhancing force the development that is crucial in cell migration. Vinculin strengthens the connections between integrins and actin filaments as they support force transfer from the cytoskeleton to the extracellular matrix (Choquet et al., 1997; Critchley et al., 1999).

It is not surprising, therefore, that we found that $\alpha$-actinin is also strongly expressed in focal adhesions, fine protrusions and around the nuclei of most migrating NCCs, and that its level is also more intense in Hcys-treated cultures, (Fig. 3, A and B and Tables 1 and 3). In addition to participating in forming focal adhesions, $\alpha$-actinin also bundles F-actin into stress fibers that migratory cells must use in contraction. This increased deployment of $\alpha$-actinin in the presence of Hcys could enhance cardiac NCCs migratory rates: the more the $\alpha$-actinin, the more the F-actin will be bound into stress fibers, and the greater the contraction capability. We also saw more spreading and irregular cell shapes in Hcys-treated cultures than controls $(p<0.025)$, (Table 2). Therefore, our evidence for increased vinculin and $\alpha$-actinin in Hcys-treated cultures suggests that the activity of the molecular switch Rho has been enhanced. Rho's downstream target proteins that affect actin organization and dynamics include vinculin and $\alpha$-actinin.

As expected, filamin, observed along the cell periphery of cultured cells showed more intense staining in the presence of Hcys as well (Fig. 3, E and F), though the difference was not significant. Interestingly, the cavities or vesicles in many cells at peripheral areas and along fine projections were more numerous and more intensely stained by anti-filamin in Hcys-treated cultures (Fig 3, F). This observation suggests that filamin may be involved in vesicular transport. Its augmentation in the presence of Hcys may be an indication of migratory cells' elevated engagement in actin polymerization, which requires a continuing renewal of G-actin precursor pools. Earlier studies have noted that vesicular traffic may also provide a continuous supply of substrate adhesion receptors that collect in adhesion plaques on the ventral surface of the lamellae. Myosin I molecules may participate in this receptor movement by transporting vesicles toward the leading edge (Ridley et al., 2003). In addition, filamin A is a ligand for the caveolae-associated protein caveolin-1 (Stahlhut \& van Deurs, 2000), a cholesterol binding integral membrane protein essential in caveolae formation, endocytosis, vesicular transport and various transduction processes (see review by Anderson, 1998). Thus the F-actin cross-linking protein, filamin also co-localizes with caveolae because it connects actin filaments to caveolar membranes. Actin polymerization at the cell periphery is important in the generation of membrane ruffles and extensions, particularly in the leading edge of a crawling cell. This activity may be expanded by establishing a pressure directed anteriorly by assembling bundles of actin together with myosin II just behind the leading lamellae. Increased local levels of filamin would support the possibility that Rac is more activated in Hcys-treated cultures than in controls. As is apparent in Figure 3, E and F, there is more actin networking in the lamellapodia of cultured Hcys-treated cardiac NCCs than in controls. Our immunolocalization evidence is consistent with the hypothesis that Hcys enhances these mechanisms.

The fourth cytoskeletal component that we examined was LIM3 protein. In cultured NCCs we found it localized mainly in nuclei and focal adhesions, with faint staining throughout the cytoplasm. Its staining intensity was more than doubled in Hcys-treated cultures compared to controls. The nuclear position of LIM3 is consistent with what other investigators have shown. LIM3 is a transcription factor that regulates transcription of ion channel genes in Drosophila embryonic neurons (Pym et al., 2006), suggesting that it has a central role in developmental processes such as axon pathfinding and synapse formation. Our results also support the microarray analysis study of Hcys-responsive genes in cardiac NCCs (Rosenquist et al., 2007) in which LIM3 expression was strongly upregulated.

We observed that LIM3 protein was additionally present in the cytoplasm in a diffuse pattern, suggesting colocalization with fine actin networks. This supports what others have shown: LIM3 is a cytoskeletal protein where it functions as an F-actin binding protein involved in actin cytoskeleton remodeling (reviewed by Khurana et al., 2002; Zheng et al., 2008). But we also observed strong LIM3 staining in the focal adhesions where tyrosine kinase, FAK, and paxillin, a substrate for FAK, all co-localize. Paxillin recruits structural and signaling molecules to focal adhesions and its LIM3 domain is critical for this (Brown et al., 1998; Saez et al., 2004). Our data show that the antibody to LIM3 that we used binds at these sites and that LIM3 and vinculin may participate 
in regulating the formation of linkages between the cytoskeleton and integrin receptors that mediate tension transmission between the actin cytoskeleton and the extracellular matrix. The relationship between LIM3 and vinculin is clearly supported in our results. Both proteins were highly expressed in cardiac NCCs, and they co-localized in focal adhesions. The effect of Hcys was to enhance this.

Because the embryonic pattern of this protein is unknown, we also used whole embryo immunolocalization of LIM3 to characterize it. We found that LIM3 is more strongly expressed in the rostral end of the head and in the heart than in other regions, though its presence was evident at lower levels near the optic cup, mandibular processes, and hyoid arches (Fig. 2). Within the heart it was possible to resolve staining that likely corresponded to the aorticopulmonary septum. Though the staining was general and clearly present in other tissues, the pattern correlates with the contributions of NCCs to these areas, and highlights the importance of LIM3 in the developing embryo. Tissues that receive NCC contributions include the cartilages, bones, cranial neurons, glia, and connective tissues of the face, the pharyngeal pouches, cornea, choroid, sclera and part of the extra-ocular muscles of the eye, the aorticopulmonary septum, and the semilunar and atrioventricular valves. It is evident that any abnormality in LIM3 in migrating cells could have serious developmental consequences in the embryo.

We observed more spread and irregular-shaped cells, and fewer rounded cells in Hcys treated cultures than in control cultures (Table 2). This could be a result of increased actin polymerization and extension activity. Although filapodia were not counted, the irregular-shaped cells had many filopodia, which are used in ruffling and adhesion activities as the cells interact with the substratum and with other cells. Other investigators have observed that Hcys increases neural crest cell motility in vitro (Brauer \& Rosenquist, 2002). For these cells to migrate, they must adhere to their substratum, but too much adhesion would reduce migration. For this reason, there is a need to strike a balance in allocating the three Ras GTPase activities in time and place for increased migration. The migratory cells were significantly more spread in Hcys-treated cultures than controls, however, the number of cell-cell attachments in Hcys-treated cultures was not different (Table 2). We saw that some NCCs moved as individuals while others migrated in clusters, as we have observed previously (Orris et al., 1999). Migrating cells share information through temporary gap junctions, allowing collective guidance and survival. Experiments with cultured cardiac NCCs from knock-out transgenic mice have shown that polarized cell movement and directional migration depend on connexin 43 (Xu et al., 2006). Our evidence suggests that Hcys has no effect on this.

In conclusion, our findings show that Hcys alters the staining patterns and intensities for actin and at least three critical proteins involved in motility, suggesting that Hcys does adjust the balance of motility organizing activity. This likely occurs via an effect on the three Ras GTPases in NCCs, and would allow greater (but not excessive) adhesion and migration. Hcys also affects the expression of LIM3 in migrating cardiac NCCs. Taken together, these findings suggest the modulation by Hcys of a signaling mechanism that involves LIM3, and that adjusts the actin-based cytoskeleton for enhanced migration. An elevation of intracellular calcium ions brought about by a Hcys-induced, inositol triphosphate-mediated release of calcium ions from the endoplasmic reticulum, as suggested by Heidenreich et al. (2008) may be part of this mechanism, particularly in mediating the necessary enhancement of adhesion. Indeed, other investigators have suggested a mechanism of regulation of Rac via calcium ions. In this mechanism calcium induces a PKC-dependent disruption of the Rac-Rho GDI complex, which promotes the translocation of $R a c$ to the plasma membrane where it can be activated by membrane-associated guanine nucleotide exchange factors (Price et al., 2003). Regardless of mechanism, the collective effect of Hcys would lead to mistimed and otherwise defective development of tissues that receive cardiac NCC contributions such as the outflow tract of the heart, and tissues where the NCCs originate.

\section{References}

Andale, V. J., Monaghan, D. T., \& Rosenquist, T. H. (1998). Dextromethorphan and other n-methyl-d- aspartate receptor antagonists are teratogenic in the avian embryo model. Pediatric Research, 43, 1-7.

Anderson, R. G. (1998). The Caveolae Membrane System. Annu Rev Biochem, 67, 199-225.

Arber, S., Hunter, J. J., Ross, J. Jr., Hongo, M., Sansig, G., Borg, J., Perriard, J. C., Chien, K. R., \& Caroni, P. (1997). MLP-deficient mice exhibit a disruption of cardiac cytoarchitectural organization, dilated cardiomyopathy, and heart failure. Cell, 88 (3), 393-403.

Bennette, G. D., Vanwaes, J., Moser, K., Chaudoin, T., Starr, L., \& Rosenquist, T. H. (2006). Failure of homocysteine to induce neural tube defects in a mouse model. Birth Defects Research Part B: Developmental and Reproductive Toxicology, 77 (2), 89-94.

Boot, M. J., Steegers-Theunissen, R. P., Poelmann, R .E., Van Iperen, L., \& Gittenberger-de Groot, A. C. (2004a). 
Homocysteine induces endothelial cell detachment and vessel wall thickening during chick embryonic development. Circulation Research, 94 (4), 542-549.

Boot, M. J., Steegers-Theunissen, R. P., Poelmann, R. E., Van Iperen, L., Lindemans, J., \& Gittenberger-de Groot, A. C. (2003). Folic acid and homocysteine affect neural crest and neuroepithelial cell growth and differentiation in vitro. Developmental Dynamics, 227 (2), 310-308.

Bostom, A. G. \& Lathrop, L. (1997). Hyperhomocysteinemia in end-stage renal disease; prevalence, etiology, and potential relationship to arteriosclerotic outcomes. Kidney International, 52 (1), 10-20.

Brauer, P. R. \& Rosenquist, T. H. (2002). Effects of elevated homocysteine on cardiac neural crest migration in vitro. Developmental Dynamics, 224, 222-230.

Brauer, P. R. \& Tierney, B. J. (2004). Consequences of elevated homocysteine during embryonic development and possible modes of action. Current Pharmaceutical Design, 10, 2719-2732.

Brown, J. C., Rosenquist, T. H., \& Monaghan, D. T. (1998). ERK2 activation by homocysteine in vascular smooth muscle cells. Biochemical and Biophysical Research Communications, 251, 669-676.

Brown, M. C., Perrotta, J. A., \& Turner, C. E. (1996). Identification of LIM3 as the principal determinant of paxillin focal adhesion localization and characterization of a novel motif on paxillin directing vinculin and focal adhesion kinase binding. Journal of Cell Biology, 135 (4), 1109-1123.

Butz, L. W. \& du Vigneaud, V. (1932). The formation of a homologue of cystine by the decomposition of methionine with sulfuric acid. Journal of Biological Chemistry, 99 (1), 125-134.

Cai, D. H. \& Brauer, P. R. (2002). Synthetic matrix metalloproteinase inhibitor decreases early cardiac neural crest migration in chicken embryos. Developmental Dynamics, 224 (4), 441-449.

Carson, N. C. J. \& Neil, D. W. (1962). Metabolic abnormalities detected in a survey of mentally backward individuals in northern ireland. Archive of Disease in Childhood, 37, 505-513.

Choquet, D., Felsenfeld, D. P., \& Sheetz, M. P. (1997). Extracellular matrix rigidity causes strengthening of integrin-cytoskeleton linkages. Cell, 88, 39-48.

Clark, E. B., (1996). Pathogenetic mechanisms of congenital cardiovascular malformations revisited. Seminars in Perinatology, 20, 465-472.

Critchley, D. R., Holt, M. R., Barry, S. T., Priddle, H., Hemmings, L., \& Norman, J. (1999). Integrin-mediated cell adhesion: The cytoskeletal connection. Biochemical Society Symposium, 65, 79-99.

Dalton, M. L., Gadson, P. F. Jr, Wrenn, R. W., \& Rosenquist, T.H. (1997). Homocysteine signal cascade: Production of phospholipids, activation of protein kinase $\mathrm{C}$, and the induction of c-fos and c-myb in smooth muscle cells. FASEB Journal, 11, 703-711.

Edqvist, P. H., Myers, S. M. \& Hallbook, F. (2006). Early identification of retinal subtypes in the developing, pre-laminated chick retina using the transcription factors Prox1, Lim1, Ap2alpha, Pax6, Is11, Is12, Lim3 and Chx10. European Journal of Histochemistry, 50 (2), 147-154.

Epeldegui, M., Pena-Melian, A., Varela-Moreiras, G., \& Perez-Miguelsanz, J. (2002). Homocysteine modifies development of neurulation and dorsal root ganglia in chick embryos. Teratology, 65(4), 171-179.

Flick, M. J. \& Konieczny, S. F. (2000). The muscle regulatory and structural protein mlp is a cytoskeletal binding partner of $\beta 1$-spectrin. Journal of Cell Science, 113, 1553-1564.

Giambernardi, T. A., Sakaguchi, A. Y., Gluhak, J., Pavlin, D., Troyer, D. A., \& Das, G. (2001). Neutrophil collagenase (MMP-8) is expressed during early development in neural crest cells as well as in adult melanoma cells. Matrix Biology, 20, 577-587.

Gilmore, A. P. \& Burridge, K. (1996). Regulation of vinculin binding to talin and action by phosphatidyl-inositol-4-5-bisphosphate. Nature, 381, 531-535.

Gourdie, R. G., Harris, B. S., Bond, J., Justus, C., Hewett, K. W., O’Brien, T. X., Thompson, R. P., \& Sedmera, D. (2003). Development of the cardiac pacemaking and conducting system. Birth Defects Research Part C: Embryo Today Reviews, 69 (1), 46-57.

Hamburger, V. \& Hamilton, H. L. (1951). A series of normal stages in the development of the chick embryo. Developmental Dynamics, 195 (4), 231-272.

Heidenreich, D. J., Reedy, M. V., \& Brauer, P. R. (2008). Homocysteine enhanced cardiac neural crest cell 
attachment in vitro by increasing intracellular calcium levels. Developmental Dynamics, 237, 2117-2128.

Humphries, J. D., Wang, P., Streuli, C., Geiger, B., Humphries, M. J., \& Ballestrem, C. (2007). Vinculin controls focal adhesion formation by direct interactions with talin and actin. Journal of Cell Biology, 179 (5), 1043-1057.

Hutson, M. R. \& Kirby, M. L. (2003). Neural crest and cardiovascular development: a 20-year perspective. Birth Defects Research Part C: Embryo Today Reviews, 69 (1), 2-13.

Kaartinen, V., Dudas, M., Nagy, A., Sridurongrit, S., Lu, M. M., \& Epstein, J. A. (2004). Cardiac outflow tract defects in mice lacking ALK2 in neural crest cells. Development, 131 (14), 3481-3490.

Kapusta, L., Haagmans, M. L., Steegers, E. A., Cuypers, M. H., Blom, H. J., \& Eskes, T. K. (1999). Congenital heart defects and maternal derangement of homocysteine metabolism. Journal of Pediatrics, 135 (6), 773-774.

Khurana, T., Khurana, B., \& Noegel, A. A. (2002). Lim proteins: association with the actin cytoskeleton. Protoplasma, 219 (1-2), 1-12.

Kirby, M. L. \& Hutson, M. R. (2010). Factors controlling cardiac neural crest cell migration. Cell Adhesion and Migration, 4 (4) 605-617.

Kirby, M. L. \& Waldo, K. L. (1995). Neural crest and cardiovascular patterning. Circulation Research, 77, 211-215.

Kirby, M. L., Gale, T. F., \& Stewart, D. E. (1983). Neural crest cells contribute to normal aorticopulmonary septation. Science, 220 (4601), 1059-1061.

Kirby, M. L., Turnage, K. L. 3rd, \& Hays, B. M. (1985). Characterization of conotruncal malformations following ablation of cardiac neural crest. Anatomical Record, 213 (1), 87-93.

Liu, G., Nellaiappan, K., \& Kagan, H.M. (1997). Irreversible inhibition of lysyl oxidase by homocysteine thiolactone and its selenium and oxygen analogues: Implications for homocystinuria. Journal of Biological Chemistry, 272, 32370-32377.

Loscalzo, J. (1996). The oxidant stress of homocysteinemia. Journal of Clinical Investigation, 98 (1), 5-7.

Maron, B. J., Olivotto, I., Spirito, P., Casey, S. A., Bellone, P., Gohman, T. E., Graham, K. J., Burton, D. A., \& Cecchi, F. (2000). Epidemiology of hypertrophic cardiomyopathy-related death: Revised in a large non-referral-based patient population. Circulation, 102 (8), 858-864.

Mills, J. L., Scott, J. M., Kirke, P. N., McPartlin, J. M., Conley, M. R., Weir, D. G., Molloy, A. M., \& Lee, Y. J. (1996). Homocysteine and neural tube defects. Journal of Nutrition, 126, 756S-760S.

Moephuli, S. R., Klein, N. W., Baldwin, M. T., \& Krider, H. M. (1997). Effects of methionine on the cytoplasmic distribution of actin and tubulin during neural tube closure in rat embryos. Procedings of the National Academy of Science USA, 94 (2), 543-548.

Nelen, W. L., Steegers, E. A., Eskes, T. K., \& Blom, H. J. (1997). Genetic risk factor for unexplained recurrent early pregnancy loss. Lancet, 350 (9081), 861.

Orris, A. S., Banicky, L. C., \&. Author (1999). Isotretinoin alters morphology, polarity, and motility of neural crest cells in culture. Reproductive Toxicology, 13 (1), 45-52.

Poelmann, R. E. \& Gittenberger-de Groot, A. C. (1999). A subpopulation of apoptosis-prone cardiac neural crest cells targets to the venous pole: multiple functions in heart development? Developmental Biology, 207, 271-286.

Poelmann, R. E., Mikawa, T., \& Gittenberger-de Groot, A. C. (1998). Neural crest cells in outflow tract septation of the embryonic chicken heart: differentiation and apoptosis. Developmental Dynamics, 212 (3), 373-384.

Pogoda, H., von der Hardt, S., Herzog, W., Kramer, C., Schwarz, H., \& Hammerschmidt, M. (2006). The proneural gene ascl1a is required for endocrine differentiation and cell survival in the zebrafish adenohypophysis. Development, 133, 1079-1089.

Price, L. S., Langeslag, M., ten Klooster, J. P., Hordijk, P. L., Jalink, K., \& Collard, J.G. (2003). Calcium signaling regulates translocation and activation of Rac. Journal of Biological Chemistry, 278 (41), 39413-39421.

Pym, E. C., Southall, T. D., Mee, C. J., Brand, A. H., \& Baines, R. A. (2006). The homeobox transcription factor even-skipped regulates acquisition of electrical properties in drosophila neurons. Neural Development, 16, 1-3.

Raposo, B., Rodriguez, C., Martinez-Gonzalez, J., \& Badimon, L. (2004). High levels of homocysteine inhibit lysyl oxidase (lox) and downregulate lox expression in vascular endothelial cells. Atherosclerosis, 177, 1-8.

Refsum, H., Nurk, E., Smith, A. D., Ueland, P. M., Gjesdal, C. G., Bjelland, I, Tverdal, A, Tell, G. S., Nygard, O., 
\& Vollset, S. E. (2006). The hordaland homocysteine study: A community-based study of homocysteine, its determinants, and associations with disease. Journal of Nutrition, 136 (6Suppl), 1731S-1740S.

Ridley, A. J., Schwartz, M. A., Burridge, K., Firtel, R. A., Ginsberg, M. H., Borisy, G., Parsons, J. T., \& Horwitz, A. R. (2003). Cell migration: Integrating signals from front to back. Science, 302 (5651), 1704-1709.

Rosenquist, T. H., Bennett, G. D., Brauer, P. R., Stewart, M. L., Chaudoin, T. R., \& Finnell, R. H. (2007). Microarray analysis of homocysteine-responsive genes in cardiac neural crest cells in vitro. Developmental Dynamics, 236, 1044-1054.

Rosenquist, T. H., Ratashak, S. A., \& Selhub, J. (1996). The use of homocysteine induces congenital defects of the heart and neural tube: Effect of folic acid. Procedings of the National Academy of Science USA, 93, 15227-15232.

Rosenquist, T. H., Schneider, A. M., \& Monaghan, D. T. (1999). N-methyl-D- aspartate receptor agonists modulate homocysteine-induced developmental abnormalities. FASEB Journal, 13, 1523-1531.

Sabapathy, K., Jochum, W., Hochedlinger, K., Chang, L., Karin, M., \& Wagner, E. F. (1999). Defective neural tube morphogenesis and altered apoptosis in the absence of both JNK1 and JNK2. Mechanisms of Development, $89,115-124$.

Saez, A. O., Zhang, W., Wu, Y., Turner, C. E., Tang, D. D., \& Gunst, S. J. (2004). Tension development during contractile stimulation of smooth muscle requires recruitment of paxillin and vinculin to the membrane. American Journal of Physiology-Cell Physiology, 286, 433-447.

Sjodal, M. \& Gunhaga, L. (2008). Expression patterns of Shh, Ptc2, Raldh3, Pitx2, Is11, Lim3 and Pax6 in the developing chick hypophyseal placode. Gene Expression Patterns, 8 (7-8), 481-485.

Snider, P., Olaopa, M., Firulli, A. B., \& Conway, S. J. (2007). Cardiovascular development and colonizing cardiac neural crest lineage. Scientific World Journal, 7, 1090-1113.

Solloway, M. J. \& Harvey, R. P. (2003). Molecular pathways in myocardial development: A stem cell perspective. Cardiovascular Research, 58, 264-277.

Stahlhut, M. \& Van Deurs, B. (2000). Identification of filamin as a novel ligand for caveolin-1-associated membrane domains by the actin cytoskeleton. Molecular Biology of the Cell, 11, 325-337.

Steegers-Theunissen, R. P., Boers, G. H., Trijbels, F. J., \& Eskes, T. K. (1991). Neural-tube defects and derangement of homocysteine metabolism. New England Journal of Medicine, 324 (3), 199-200.

Tierney, B. J., Ho, T., Reedy, M. V., \& Brauer, P. R. (2004). Homocysteine inhibits cardiac neural crest cell formation and morphogenesis. Developmental Dynamics, 229, 63-73.

Wang, X. (1999). A theory for the mechanism of homocysteine-induced vascular pathogenesis. Medical Hypotheses, 53 (5), 386-394.

Weekes, J., Barry, S. T., \& Critchley, D. R. (1996). Acidic phospholipids inhibit the intramolecular association between the $\mathrm{n}$ - and c-terminal regions of vinculin, exposing actin-binding and protein kinase c phosphorylation sites. Biochemical Journal, 314, 827-832.

Xu, X., Francis, R., Wei, C.J., Linask, K. L., \& Lo, C.W. (2006). Connexin 43-mediated modulation of polarized cell movement and the directional migration of cardiac neural crest cells. Development, 133, 3629-3639.

Yang, Z.Z. \& Zou, A.P. (2003). Homocysteine Enhances TIMP-1 Expression and Cell Proliferation Associated with NADH Oxidase in Rat Mesangial Cells. Kidney International, 63, 1012-1020.

Zheng, B., Wen, J. K., \& Han, M. (2008). HhLIM is a novel f-actin binding protein involved in actin cytoskeleton remodeling. FEBS Journal, 275 (7), 1568-1578. 
Table 1. Quantitation of antibody staining following color thresholding

\begin{tabular}{|c|c|c|c|c|}
\hline & LIM3 & $\alpha$-actinin & Vinculin & Filamin A \\
\hline Control & $50.2 \pm 18.2$ & $67.6 \pm 24.7$ & $66.5 \pm 45.3$ & $47.6 \pm 35.0$ \\
\hline Hcys $(100 \mu \mathrm{M})$ & $116.0^{*} \pm 87.8$ & $119.7^{*} \pm 60.3$ & $149.2^{*} \pm 83.3$ & $83.8 \pm 35.2$ \\
\% Increase & 131 & 77 & 125 & 76 \\
T-test & $\mathrm{p}<0.026$ & $\mathrm{p}<0.011$ & $\mathrm{p}<0.002$ & $\mathrm{p}<0.156$ \\
\hline
\end{tabular}

Data represent means and standard errors of areas with red immunostain above threshold $\left(\mu \mathrm{m}^{2} \mathrm{X} 1000\right)$ in cultured cardiac NCCs from stages 10-13 neural tube explants. The staining revealed the levels of the indicated proteins. There were 10-13 explants per group and at least 100 cells were sampled from each explant.

*Significantly greater than control.

Table 2. Cell morphology and cell-cell attachment pattern

\begin{tabular}{|c|c|c|c|}
\hline & $\begin{array}{c}\text { Mean percent of spread, } \\
\text { irregular-shaped cells } \pm \mathrm{SD}\end{array}$ & $\begin{array}{c}\text { Mean percent of round } \\
\text { cells } \pm \mathrm{SD}\end{array}$ & $\begin{array}{c}\text { Mean percent of cells } \\
\text { having at least one } \\
\text { attachment } \pm \text { SD }\end{array}$ \\
\hline Control & $75 \pm 10$ & $25 \pm 10$ & $86 \pm 8$ \\
\hline Hcys & $83+8$ & $17+6$ & $88+7$ \\
\hline Percent change & 11 & -32 & 2 \\
\hline T-test & $\mathrm{p}<0.025$ & $\mathrm{p}<0.001$ & $\mathrm{p}<0.989$ \\
\hline
\end{tabular}

Data represent cell morphology and cell-cell attachment patterns for cultured cardiac NCCs from neural tube explants dissected from stage 10-13 chick embryos. $\mathrm{N}=10$ explants per group.

Table 3. Summary of immunolocalization and functions of $\alpha$-actinin, vinculin, filamin and LIM3 proteins in migrating cardiac NCCs

\begin{tabular}{|l|l|l|l|}
\hline Protein & Protein function & $\underline{\underline{\text { Immunolocalization in cardiac }}}$ & Ras GTPase control \\
\hline Alpha-actinin & $\begin{array}{l}\text { Bundles actin filament into } \\
\text { stress fibers }\end{array}$ & $\begin{array}{l}\text { Around nucleus, focal } \\
\text { adhesions, filopodia }\end{array}$ & Rho \\
\hline Vinculin & $\begin{array}{l}\text { Links actin stress fibers to } \\
\text { integrins }\end{array}$ & Focal adhesions, lamellipodia & $\begin{array}{l}\text { Rho (for focal } \\
\text { ad-hesions) Rac (for } \\
\text { focal adhesions in } \\
\text { lamelli-podia) }\end{array}$ \\
\hline Filamin & $\begin{array}{l}\text { Creates networks by } \\
\text { cross-linking actin filaments } \\
\text { in leading lamellae }\end{array}$ & $\begin{array}{l}\text { Cell periphery, filopodia, } \\
\text { caveolae, trans-Golgi vesicles }\end{array}$ & Rac \\
\hline LIM3 & $\begin{array}{l}\text { Transcription factor in } \\
\text { cardiogenesis; actin filament } \\
\text { binding protein }\end{array}$ & $\begin{array}{l}\text { Nucleus, focal adhesions, } \\
\text { faintly throughout cytoplasm }\end{array}$ & Rho for focal adhesions \\
\hline
\end{tabular}



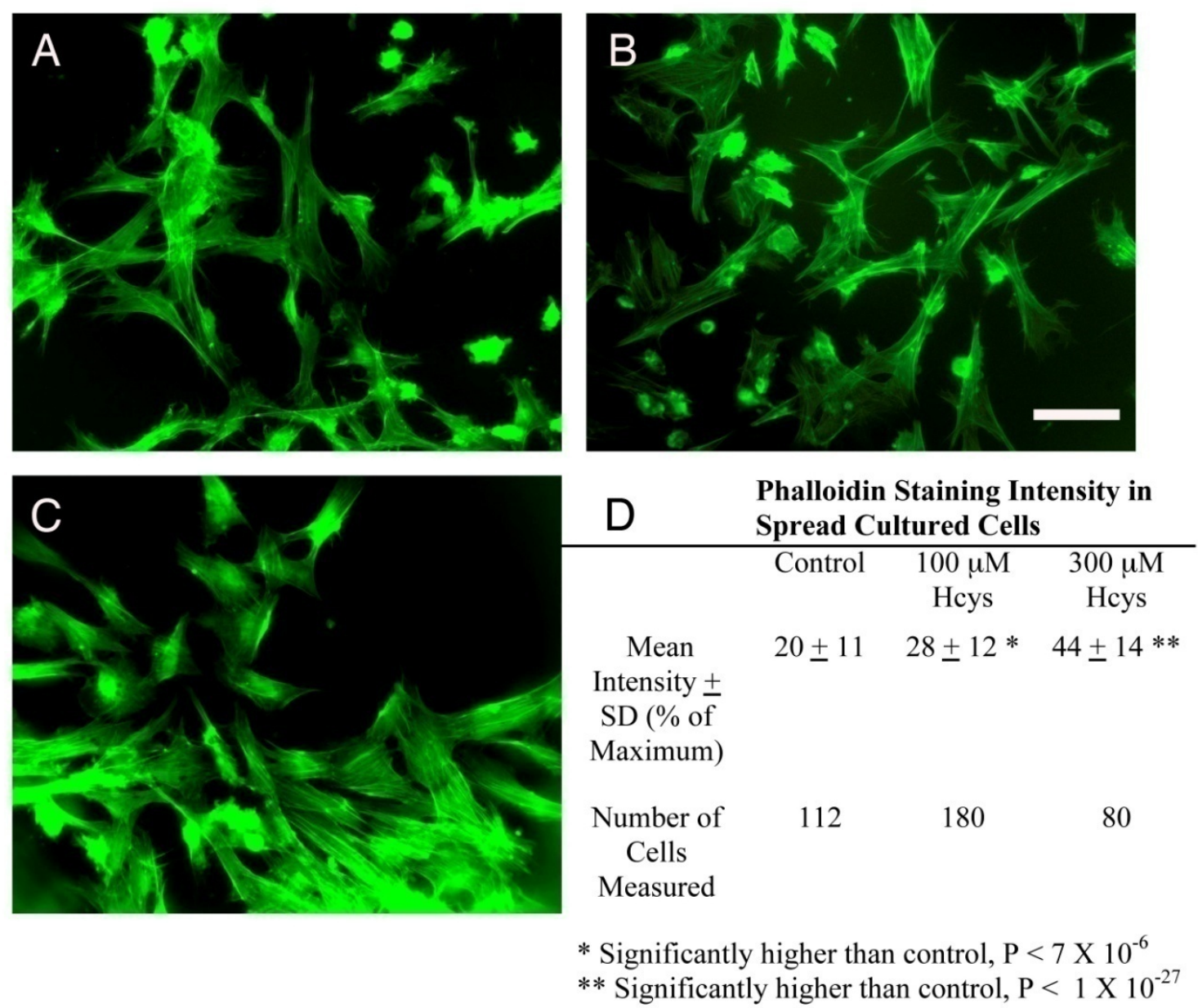

Figure 1. Homocysteine augments stress fibers: Actin localization and quantitation in phalloidin-stained, cultured cardiac neural crest cells. A, Cells cultured in the absence of Hcys. Stress fibers are very fine. B, Cells cultured in $100 \mu \mathrm{M}$ Hcys. Stress fibers are brighter and have greater apparent thickness. C, Cells cultured in $300 \mu \mathrm{M}$ Hcys. Stress fibers are thicker and even more intense. Bar in $B=50 \mu \mathrm{m}$. 

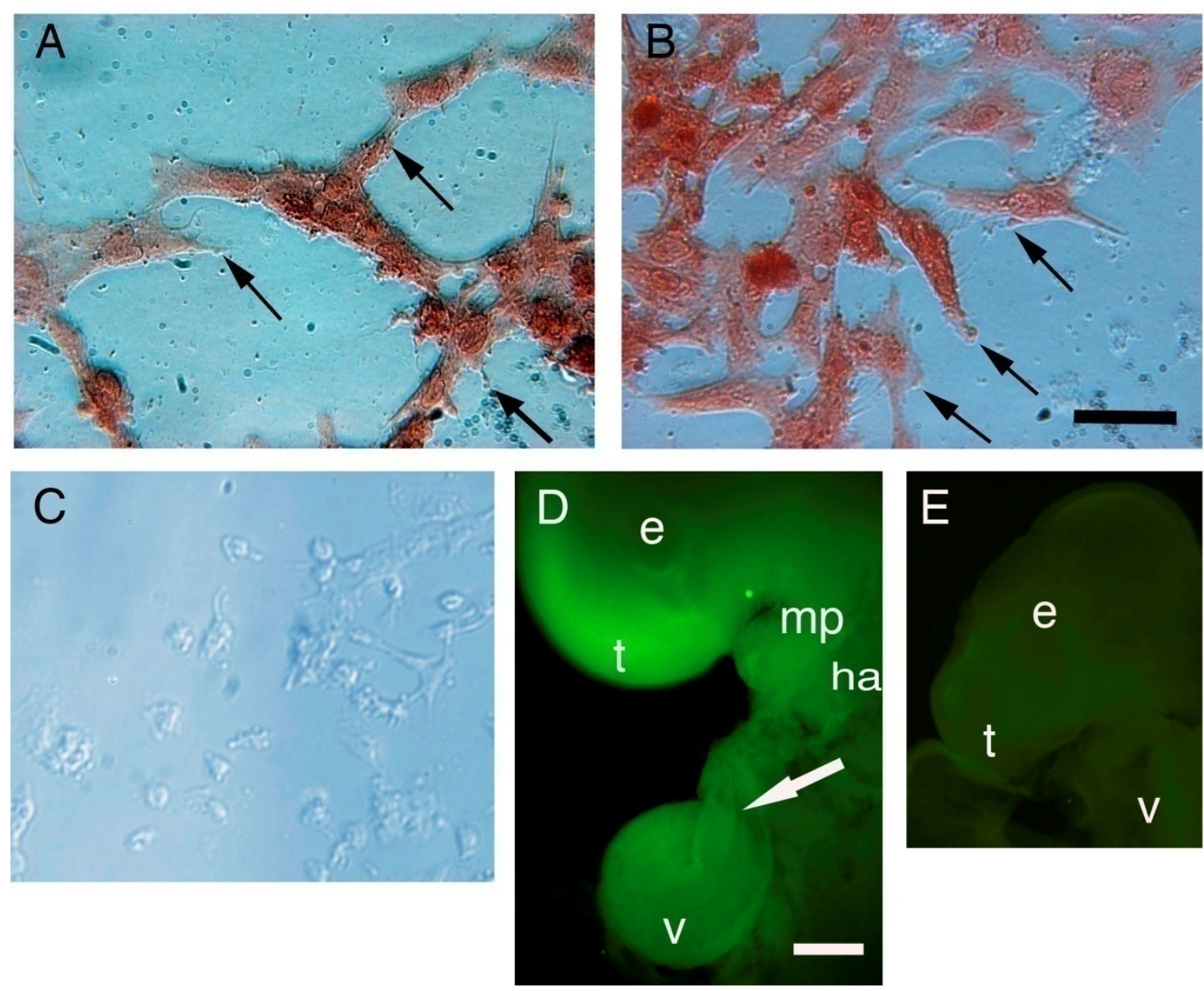

Figure 2. Localization of LIM3 protein in cultured cardiac neural crest cells and in embryos. A, untreated neural crest cells immunostained for LIM3. The protein is evident in nuclei, focal adhesions (arrows) and throughout the cytoplasm. B, Hcys-treated cells. LIM3 is seen in the same places but is somewhat more intense. In both images there are rounded cells lying on the more spread cells. These rounded cells are vividly red because of their more three-dimensional shape. C, Immunostaining negative control using no primary antibody. D, Whole embryo at stage 16 immunostained for LIM3 protein and detected with Alexafluor-conjugated secondary antibodies. The anterior-most brain segment and the heart, particularly the aorticopulmonary septum (arrow) stain intensely whereas the mandibular process and hyoid arch stain moderately. E, Whole stage 16 embryo immunostaining negative control which shows only background staining. Bar in $\mathrm{B}=50 \mu \mathrm{m}$; bar in $\mathrm{D}=400 \mu \mathrm{m}$. e, eye; ha, hyoid arch; mp, mandibular process; t, telencephalon; v, ventricle. 

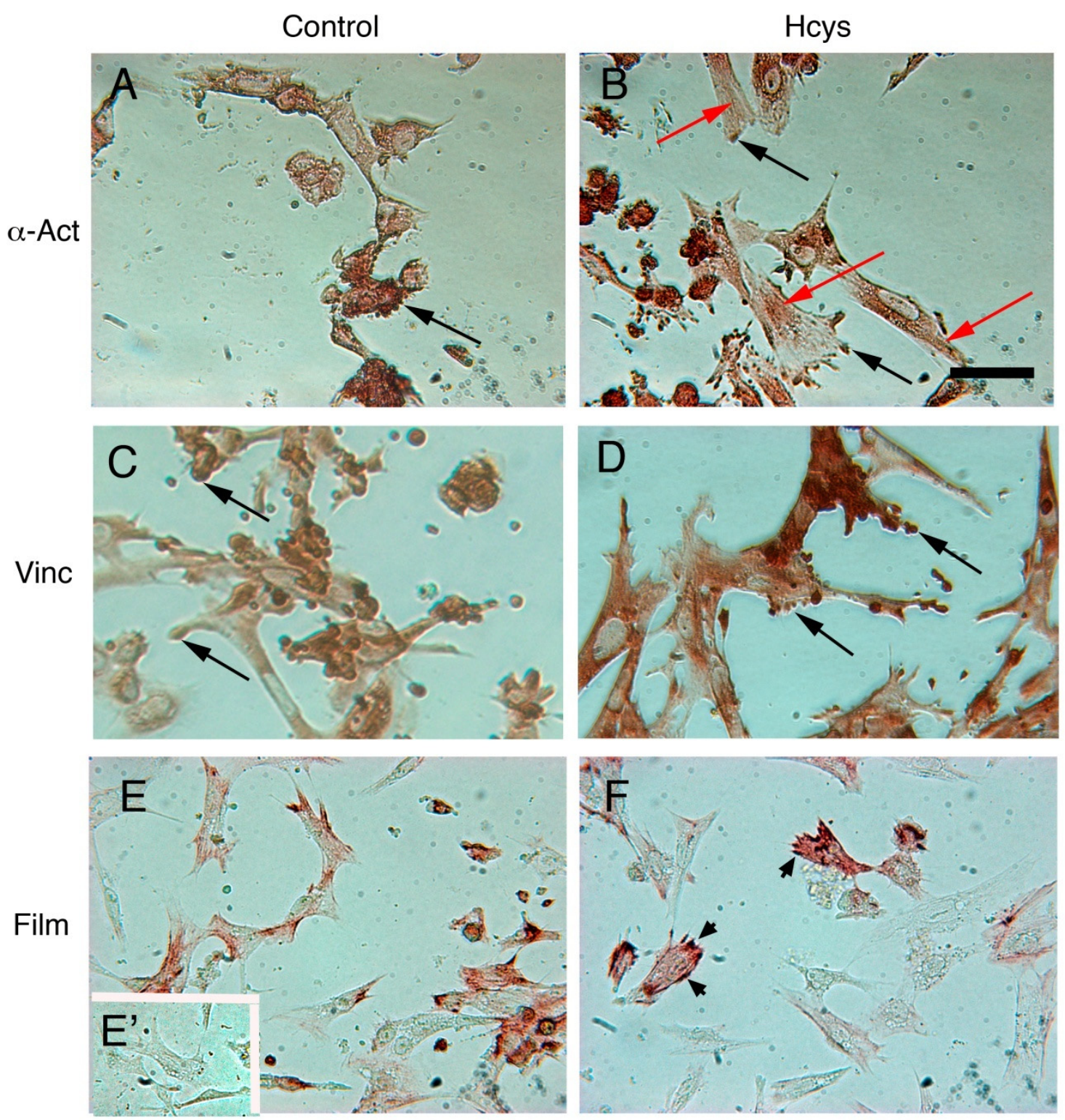

Figure 3. Localization of $\alpha$-actinin, vinculin, and filamin in migrating neural crest cells. Homocysteine enhanced focal adhesions and stress fibers. A, C, and E, untreated cells immunostained with antibodies to $\alpha$-actinin (A), vinculin (C) and filamin (E). E' inset, filamin immunostaining negative control using no primary antibody. The negative controls for immunostaining with other antibodies looked very similar showing low background staining. B, D, and F, Hcys treated cells immunostained with antibodies to $\alpha$-actinin (B), vinculin (D) and filamin (F). Black arrows indicate focal adhesions and red arrows indicate stress fibers, which were both more intense in treated cells. Antibodies to filamin stained cytoplasm along the periphery of the cultured cells where actin networks likely reside, and was less intense in flat, migrating cells. Some cells that stained intensely with anti-filamin showed stained structures at the cell periphery that appeared to be caveolae (arrowheads in F) with trans-Golgi vesicles nearby. Bar in B is $50 \mu \mathrm{m}$ and scales all seven images. 